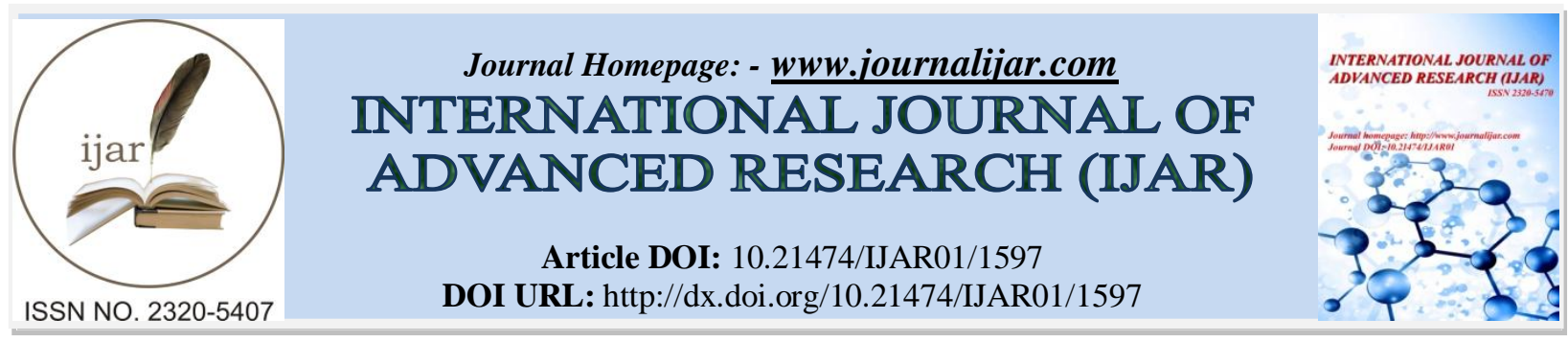

RESEARCH ARTICLE

\title{
ORTHODONTIC TREATMENT ATTITUDE AND COOPERATION: A PROSPECTIVE STUDY OF PATIENTS' AND PARENTS' RESPONSES IN CHINESE POPULATION.
}

Acharya $A^{1}$, Varun Pratap Singh ${ }^{2}$, Giri D3, Bai Y $M^{4}$, Zhang $Z X^{5}$, Hu L A ${ }^{6}$, Chen $\mathrm{S}^{7}$ and Jing $\mathrm{M}^{4}$.

1. Department of Orthodontics, Nobel Medical College and Teaching Hospital, Biratnagar, Nepal.

2. Specialist Orthodontist, Al Ain Dental Center(SEHA-AHS), AlAin, Abu Dhabi, United Arab Emirates.

3. Department of Periodontics, Nobel Medical College and Teaching Hospital, Biratnagar, Nepal.

4. Department of Orthodontics, Tongji Hospital, Huazhong University of Science and Technology, Wuhan, China.

\section{Manuscript Info}

[.......................

Manuscript History

Received: 12 July 2016

Final Accepted: 13 August 2016

Published: September 2016

Key words:-

orthodontic treatment; attitude;

cooperation; Chinese population

\section{Abstract}

Introduction:- The final outcome of an orthodontic treatment depends on the patients' compliance in following orthodontist's instructions.

Objectives:- To explore the association between orthodontic patients' and their parents' response on orthodontic treatment attitude with the patients' response regarding treatment cooperation. Second, to investigate the change in treatment attitude and cooperation response when they have undergone 6 months of active treatment.

Methods:- Data were collected from 79 orthodontic patients undergoing fixed orthodontic treatment (36.7\% boys and $63.3 \%$ girls), and their parents. Responses were collected once during appointment of Diagnosis and Treatment Plan and finally after 6 months of active treatment.

Results:- Patients' level of treatment attitude was significantly correlated with their level of treatment cooperation. After 6 months of treatment the patients' reported greater levels of treatment attitude as compared to when they were at the beginning of treatment. Pretreatment parents' attitude responses were not significantly correlated with the children's actual cooperation responses.

Conclusion:- Patients' attitude was found to influence their cooperative behavior, so orthodontists' efforts to generate positive attitude towards the treatment, especially in patients, would guide the clinician to carry out an efficient treatment in optimal time.

Copy Right, IJAR, 2016,. All rights reserved.

An orthodontist notices the patients who follow her instructions regarding using their headgear, maintaining their oral hygiene, and showing up for their regularly scheduled appointments get remarkably better final results than the patients who are less fastidious, or less compliant, in following her order ${ }^{1}$. It's of utmost concern for orthodontists to make their patients motivated towards the treatment and, hence, obtain much cooperation so as to finish the lengthy treatment in minimal time. Several researchers and psychologists have proved that positive attitude and optimism will ultimately lead to success.

Corresponding Author:- Acharya A.

Address:- Department of Orthodontics, Nobel Medical College and Teaching Hospital, Biratnagar, 
Factors that might significantly predict an orthodontic patient's cooperation with treatment include the patient's attitude toward the treatment immediately before and during the treatment and the ability to cope with any discomfort during treatment ${ }^{2,3}$. The orthodontist's behavior influences patient satisfaction, the orthodontist-patient relationship and patient cooperation ${ }^{4}$. Positive reinforcement by praising patients verbally when they show good cooperation is generally used in routine clinical practice ${ }^{5}$.

Our objectives were to evaluate association between treatment attitude as responded by patients and their parents with the patients' reported orthodontic treatment cooperation. Andto compare orthodontic treatment attitude of child and adolescent patients and their parents at an initial diagnostic appointment with their responses after 6 months of treatment.

\section{Methods:-}

Data were collected from 79 orthodontic patients ( 29 boys, 50 girls) who had undergone orthodontic treatment and their parents or legal guardians at Department of Orthodontics, TongjiHospital, Huazhong University of Science and Technology, Hubei, Central China. Institutional permission was obtained from the Institutional Review Board of Tongji Hospital. The inclusion criteria were: 1) Patients aged 11-19 yrs(average 14.2years; SD=2.47)accompanied by their parents or legal guardians in Diagnosis and Treatment Plan appointment, 2) the patients gave written assent and/or parents gave written consent, 3)the patients and parents could read and understand Mandarin, 4) the patients having orthodontic treatment had fixed attachments or braces at least on their anterior teeth.

Out of 79 patients 52 patients $(65.8 \%)$ had undergone Non Extraction orthodontic treatment and remaining 27 patients $(34.2 \%)$ had undergone Extraction orthodontic treatment.In this study all the subjects of known ethnicity are Han. Of 79 parents or legal guardians $30(37.9 \%)$ were men and 49(62.02\%) were womenat the Diagnosis and Treatment Plan appointment where as $33(41.77 \%)$ men and 46(58.22\%) women after 6 months of treatment.Participants were recruited by graduate students in the department. The patients did not have any orthodontic treatment before.

Patients and parents at the beginning of orthodontic treatment answered the questionnaire "To be Orthodontic Patient Survey" and "To be Orthodontic Parent Survey" and after 6 months of active treatment answered the questionnaire "Current Orthodontic Patient Survey" and "Current Orthodontic Parent Survey" respectively. The questionnaire for patients and parents included 1) Age, gender and race 2) Orthodontic TreatmentAttitude, and 3) Orthodontic Treatment Cooperation.Translation of the items was done with the help of an expert.The patients' Orthodontic Treatment Attitude questionnaire has reliability of 0.78 where as patients' Orthodontic Treatment Cooperationquestionnaire has reliability of 0.81 . Similarly, reliability of parents' Orthodontic Treatment Attitude questionnairewas 0.72 and parents' Orthodontic Treatment Cooperation questionnaire was 0.81 . Reliability was given in Cronbach's alpha based on the results of Pilot study. Face and construct validity was given based on expert opinions.Items in Orthodontic Treatment Cooperation Questionnaire were based on Adams et al,where as items regarding Orthodontic Treatment Attitude was based on clinical experience and past literature.

The data were analyzed with SPSS software (version18.0). The hypothesis regarding association between treatment attitude and treatment cooperation; pre treatment and during treatment attitude levels of the child and adolescent patients; and association between parents' and their children's responses, all were tested with Pearson Correlation Analysis.Paired Sample $T$ test was performed to calculate the variation of attitude before treatment and during treatment between different genders in parents.

\section{Results:-}

All answers for the Orthodontic Treatment Attitude and Orthodontic Treatment Cooperation items were given on 5point rating scales from 1, "disagree strongly," to 5, "agree strongly."

As a whole, at the appointment of Diagnosis and Treatment plan, patients' attitude was not significantly correlated with the parents' attitude for their children's treatment. Whereas after 6months of active treatment parents had significantly higher level of treatment attitude in general as compared to the child patients, the items for evaluation of attitude of parents before treatment (item 1 through item 5) mean19.59 SD=2.33 Vs 19.38 SD 2.79. $\mathrm{r}=.279$, $\mathrm{P}=0.013$. 
Child and adolescent patients after 6 months of treatment reported greater levels of treatment attitude as compared to their levels of treatment attitude at the beginning of treatment. Mean 19.38, SD2.79 vs. mean 19.16, SD 2.67, P< 0.001. Similarly, patients during treatment reported they would be less likely to cooperate with accessories of treatment methods as per orthodontist's instructions, as compared to when they were at the beginning of the treatment: mean 20.71, SD 1.75 vs. mean 21.34,SD 2.45, $\mathrm{P}<0.001$.

Patients' response on the item "I like to have/want braces on my teeth" had significant negative correlation with the parents' response on "I like to have braces on my child's teeth" with the correlation coefficient of -.282, P=0.013. This was significant particularly when the child patients were at the beginning of the treatment. (Table I)

Child and adolescent patients' levels of treatment attitude as a whole were significantly correlated with their levels of treatment cooperation in general.558, $\mathrm{P}<.001$ pretreatment; .482, $\mathrm{P}<.001$ during treatment (Table II and III). However during treatment patients' attitude response as a whole were not correlated significantly with their response on cooperation in wearing bite plate as instructed (Table III). Parents' attitude during treatment was correlated significantly with the patients' response to cooperate in the treatment $.226, \mathrm{P}=.045$ where as it was not correlated significantly before the treatment. Child patients' response regarding treatment attitude was correlated significantly with their parents' response that their child would cooperate with the treatment recommendations $.225, \mathrm{P}=.046$ (pretreatment); .341, $\mathrm{P}=.002$ (during treatment).

Concerning the parents' levels of treatment attitude for their children to have braces and their perceptions of their children's cooperation in the treatment, several significant relationships werefound. In general, the higher the level of treatment attitude for their children's treatment, the more they agreed that their children would strictly follow orthodontist's instructions and accept accessory treatment methods such as wearing headgear, bite plate, elastic bands for perfect orthodontic outcome(.606,P $<.001$ pretreatment; .461,P<.001during treatment).

Table I:- Correlations between child patients' and parents' responses concerning Orthodontic Treatment Attitude before treatment and during treatment

\begin{tabular}{|c|c|c|c|c|c|c|}
\hline \multirow[t]{2}{*}{ CHILD PATIENTS } & \multicolumn{6}{|l|}{ PARENTS } \\
\hline & $\begin{array}{l}\text { Type of } \\
\text { appointment }\end{array}$ & $\begin{array}{l}\text { Item 1: I am } \\
\text { in favor of } \\
\text { orthodontic } \\
\text { treatment of } \\
\text { my child }\end{array}$ & $\begin{array}{l}\text { Item 2: I am } \\
\text { willing to get } \\
\text { orthodontic } \\
\text { treatment of } \\
\text { my child }\end{array}$ & $\begin{array}{l}\text { Item } 3: M y \\
\text { child can } \\
\text { tolerate the } \\
\text { discomfort } \\
\text { during the } \\
\text { treatment }\end{array}$ & $\begin{array}{l}\text { Item4:I like to } \\
\text { have braces } \\
\text { on my child's } \\
\text { teeth }\end{array}$ & $\begin{array}{l}\text { Item5: It is } \\
\text { necessary for } \\
\text { my child to } \\
\text { have braces }\end{array}$ \\
\hline \multirow{2}{*}{$\begin{array}{l}\text { Item 1. I am in favor } \\
\text { of orthodontic } \\
\text { treatment }\end{array}$} & $\begin{array}{l}\text { Before } \\
\text { Treatment }\end{array}$ & .129 & .040 & .043 & .025 & .120 \\
\hline & $\begin{array}{l}\text { During } \\
\text { Treatment }\end{array}$ & .212 & .216 & .053 & .061 & .026 \\
\hline \multirow{2}{*}{$\begin{array}{l}\text { Item } 2 . \text { I am willing to } \\
\text { get orthodontic } \\
\text { treatment }\end{array}$} & $\begin{array}{l}\text { Before } \\
\text { Treatment }\end{array}$ & .158 & .096 & .110 & .001 & -.005 \\
\hline & $\begin{array}{l}\text { During } \\
\text { Treatment }\end{array}$ & .213 & $\begin{array}{l}.231 \\
P=0.042\end{array}$ & .159 & .149 & -.002 \\
\hline \multirow{2}{*}{$\begin{array}{l}\text { Item3.I can tolerate } \\
\text { the discomfort during } \\
\text { the treatment }\end{array}$} & $\begin{array}{l}\text { Before } \\
\text { Treatment }\end{array}$ & .181 & .098 & .157 & .009 & .057 \\
\hline & $\begin{array}{l}\text { During } \\
\text { Treatment }\end{array}$ & .155 & .179 & $\begin{array}{l}.254 \\
P=0.024\end{array}$ & .082 & .124 \\
\hline \multirow{2}{*}{$\begin{array}{l}\text { Item4: I like to have } \\
\text { braces on my teeth }\end{array}$} & $\begin{array}{l}\text { Before } \\
\text { Treatment }\end{array}$ & .147 & .044 & -.031 & $\begin{array}{l}-.282 \\
P=.013\end{array}$ & -.044 \\
\hline & $\begin{array}{l}\text { During } \\
\text { Treatment }\end{array}$ & $\begin{array}{l}.262 \\
P=0.021\end{array}$ & $\begin{array}{l}.223 \\
P=0.050\end{array}$ & .103 & .138 & -.014 \\
\hline \multirow[t]{2}{*}{$\begin{array}{l}\text { Item5: It is necessary } \\
\text { for me to have braces }\end{array}$} & $\begin{array}{l}\text { Before } \\
\text { Treatment }\end{array}$ & .211 & .028 & .023 & .025 & .179 \\
\hline & $\begin{array}{l}\text { During } \\
\text { Treatment }\end{array}$ & $\begin{array}{l}.273 \\
P=0.016\end{array}$ & .186 & .122 & .169 & .145 \\
\hline
\end{tabular}


Table II:- Correlation between child patients' and parents' responses concerning treatment attitude and treatment cooperation (Diagnosis and treatment plan appointment).

\begin{tabular}{|c|c|c|c|c|c|c|c|c|c|c|}
\hline \multirow[b]{2}{*}{$\begin{array}{l}\text { Child } \\
\text { response } \\
\text { concernin } \\
\text { g } \\
\text { treatment } \\
\text { cooperati } \\
\text { on }\end{array}$} & \multicolumn{5}{|c|}{ Child response concerning treatment attitude } & \multicolumn{5}{|c|}{$\begin{array}{l}\text { Parents response concerning treatment } \\
\text { attitude }\end{array}$} \\
\hline & $\begin{array}{l}\text { Item 1:I } \\
\text { am in } \\
\text { favor of } \\
\text { orthodo } \\
\text { ntic } \\
\text { treatmen } \\
t\end{array}$ & $\begin{array}{l}\text { Item } 2: I \\
\text { am } \\
\text { willing } \\
\text { to get } \\
\text { Orthodo } \\
\text { ntic } \\
\text { treatmen } \\
t\end{array}$ & $\begin{array}{l}\text { Item 3:I } \\
\text { can } \\
\text { tolerate } \\
\text { the } \\
\text { discomf } \\
\text { ort } \\
\text { during } \\
\text { the } \\
\text { treatme } \\
\text { nt }\end{array}$ & $\begin{array}{l}\text { Item } \\
4: I \\
\text { like } \\
\text { to } \\
\text { have } \\
\text { brac } \\
\text { es on } \\
\text { my } \\
\text { teeth }\end{array}$ & $\begin{array}{l}\text { Item 5: } \\
\text { It is } \\
\text { necess } \\
\text { ary for } \\
\text { me to } \\
\text { have } \\
\text { braces }\end{array}$ & $\begin{array}{l}\text { Item 1:I } \\
\text { am in } \\
\text { favor of } \\
\text { orthodo } \\
\text { ntic } \\
\text { treatmen } \\
\text { t of my } \\
\text { child }\end{array}$ & $\begin{array}{l}\text { Item } 2: I \\
\text { am } \\
\text { willing } \\
\text { to get } \\
\text { orthodo } \\
\text { ntic } \\
\text { treatmen } \\
\text { t of my } \\
\text { child }\end{array}$ & $\begin{array}{l}\text { Item 3: } \\
\text { My } \\
\text { child } \\
\text { can } \\
\text { tolerate } \\
\text { the } \\
\text { discomf } \\
\text { ort } \\
\text { during } \\
\text { the } \\
\text { treatme } \\
\text { nt }\end{array}$ & $\begin{array}{l}\text { Item } \\
4: \text { I } \\
\text { like } \\
\text { to } \\
\text { have } \\
\text { brac } \\
\text { es on } \\
\text { my } \\
\text { child } \\
\text { 's } \\
\text { teeth }\end{array}$ & $\begin{array}{l}\text { Item } \\
\text { 5:It is } \\
\text { necess } \\
\text { ary for } \\
\text { my } \\
\text { child to } \\
\text { have } \\
\text { braces }\end{array}$ \\
\hline $\begin{array}{l}\text { I will } \\
\text { strictly } \\
\text { follow } \\
\text { orthodonti } \\
\text { sts } \\
\text { instruction } \\
\text { s }\end{array}$ & $\begin{array}{l}.422 \\
\mathrm{P}<.01\end{array}$ & $\begin{array}{l}.293 \\
\text { P } .009\end{array}$ & $\begin{array}{l}.359 \\
\text { P } .001\end{array}$ & .168 & $\begin{array}{c}.363 \\
\text { P.001 }\end{array}$ & -.088 & -.031 & .082 & -.067 & -.015 \\
\hline $\begin{array}{l}\text { If } \\
\text { orthodonti } \\
\text { sts asked } \\
\text { me to } \\
\text { wear } \\
\text { headgear I } \\
\text { will do as } \\
\text { instructed }\end{array}$ & $\begin{array}{c}.338 \\
\text { P } .002\end{array}$ & $\begin{array}{l}.402 \\
\mathrm{P}<.01\end{array}$ & $\begin{array}{l}.437 \\
\mathrm{P}<.01\end{array}$ & $\begin{array}{c}.243 \\
\mathrm{P} \\
.031\end{array}$ & $\begin{array}{l}.368 \\
\mathrm{P}<.01\end{array}$ & -.092 & -.088 & .204 & .054 & -.008 \\
\hline $\begin{array}{l}\text { If } \\
\text { orthodonti } \\
\text { sts asked } \\
\text { me to } \\
\text { wear } \\
\text { biteplate I } \\
\text { will do as } \\
\text { instructed }\end{array}$ & $\begin{array}{l}.346 \\
\text { P } .002\end{array}$ & $\begin{array}{l}.429 \\
\mathrm{P}<.01\end{array}$ & $\begin{array}{l}.360 \\
\text { P } .001\end{array}$ & $\begin{array}{c}.267 \\
\mathrm{P} \\
.017\end{array}$ & $\begin{array}{l}.389 \\
\mathrm{P}<.01\end{array}$ & -.022 & .071 & .150 & -.106 & .061 \\
\hline $\begin{array}{l}\text { If } \\
\text { orthodonti } \\
\text { sts asked } \\
\text { me to } \\
\text { wear } \\
\text { retainers I } \\
\text { will do as } \\
\text { instructed }\end{array}$ & $\begin{array}{l}.294 \\
\text { P } .008\end{array}$ & $\begin{array}{l}.246 \\
\text { P } .029\end{array}$ & $\begin{array}{l}.292 \\
\text { P } .009\end{array}$ & $\begin{array}{c}.223 \\
\mathrm{P} \\
.048\end{array}$ & $\begin{array}{l}.397 \\
\mathrm{P}<.01\end{array}$ & .010 & .036 & .006 & -.212 & -.048 \\
\hline $\begin{array}{l}\text { If } \\
\text { orthodonti } \\
\text { sts asked } \\
\text { me to } \\
\text { wear } \\
\text { elastics I } \\
\text { will do as } \\
\text { instructed }\end{array}$ & $\begin{array}{l}.407 \\
\mathrm{P}<.01\end{array}$ & $\begin{array}{l}.294 \\
\text { P .009 }\end{array}$ & $\begin{array}{l}.315 \\
\text { P } .005\end{array}$ & .207 & $\begin{array}{c}.412 \\
\mathrm{P}<.01\end{array}$ & -.004 & -.02 & .027 & -.089 & -.022 \\
\hline
\end{tabular}




\begin{tabular}{|c|c|c|c|c|c|c|c|c|c|c|}
\hline 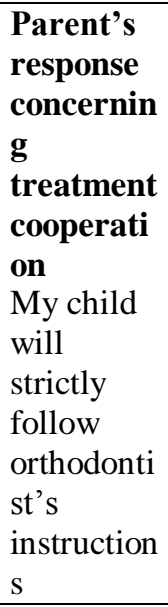 & .158 & $\begin{array}{c}.358 \\
\text { P } .001\end{array}$ & .188 & .092 & .105 & .196 & .206 & .149 & .201 & .151 \\
\hline $\begin{array}{l}\text { If } \\
\text { orthodonti } \\
\text { st asked } \\
\text { to wear } \\
\text { headgear } \\
\text { my child } \\
\text { will do as } \\
\text { instructed }\end{array}$ & .140 & $\begin{array}{c}.265 \\
\text { P } .018\end{array}$ & $\begin{array}{c}.262 \\
\text { P .019 }\end{array}$ & .159 & .108 & $\begin{array}{c}.291 \\
\text { P } .009\end{array}$ & $\begin{array}{c}.272 \\
\text { P.015 }\end{array}$ & $\begin{array}{c}.314 \\
\text { P.005 }\end{array}$ & .220 & $\begin{array}{c}.339 \\
\text { P .002 }\end{array}$ \\
\hline $\begin{array}{l}\text { If } \\
\text { orthodonti } \\
\text { st asked } \\
\text { to wear } \\
\text { biteplate } \\
\text { my child } \\
\text { will do as } \\
\text { instructed }\end{array}$ & .002 & .200 & .206 & .199 & -.023 & $\begin{array}{c}.322 \\
\text { P.004 }\end{array}$ & $\begin{array}{c}.395 \\
P<.01\end{array}$ & $\begin{array}{c}.377 \\
P<.01\end{array}$ & $\begin{array}{l}.393 \\
\mathrm{P} \\
<.01\end{array}$ & $\begin{array}{c}.363 \\
\text { P .001 }\end{array}$ \\
\hline $\begin{array}{l}\text { If } \\
\text { orthodonti } \\
\text { st ask to } \\
\text { wear } \\
\text { retainers } \\
\text { my child } \\
\text { will do as } \\
\text { instructed }\end{array}$ & -.006 & .159 & . 124 & .105 & .037 & .117 & $\begin{array}{c}.237 \\
\text { P } .036\end{array}$ & $\begin{array}{c}.225 \\
\text { P } .046\end{array}$ & $\begin{array}{c}.330 \\
\mathrm{P} \\
.003\end{array}$ & $\begin{array}{c}.306 \\
\text { P.006 }\end{array}$ \\
\hline $\begin{array}{l}\text { If } \\
\text { orthodonti } \\
\text { st asked } \\
\text { to wear } \\
\text { elastics } \\
\text { my child } \\
\text { will do as } \\
\text { instructed }\end{array}$ & .108 & .124 & .200 & .095 & .021 & $\begin{array}{c}.312 \\
\text { P.005 }\end{array}$ & $\begin{array}{c}.322 \\
\text { P.004 }\end{array}$ & $\begin{array}{c}.313 \\
\text { P.005 }\end{array}$ & $\begin{array}{c}.401 \\
\mathrm{P} \\
<.01\end{array}$ & $\begin{array}{c}.450 \\
\mathrm{P}<.01\end{array}$ \\
\hline
\end{tabular}


Table III:- Correlation between child patients' and parents' responses concerning treatment attitude and treatment cooperation (At 6months of Treatment).

\begin{tabular}{|c|c|c|c|c|c|c|c|c|c|c|}
\hline \multirow[b]{2}{*}{$\begin{array}{l}\text { Child } \\
\text { responses } \\
\text { concernin } \\
\text { g } \\
\text { treatment } \\
\text { cooperati } \\
\text { on }\end{array}$} & \multicolumn{5}{|c|}{ Child responses concerning treatment attitude } & \multicolumn{5}{|c|}{$\begin{array}{l}\text { Parent responses concerning treatment } \\
\text { attitude }\end{array}$} \\
\hline & $\begin{array}{l}\text { Item 1:I } \\
\text { am in } \\
\text { favor of } \\
\text { orthodo } \\
\text { ntic } \\
\text { treatmen } \\
t\end{array}$ & $\begin{array}{l}\text { Item 2:I } \\
\text { am } \\
\text { willing } \\
\text { to get } \\
\text { Orthodo } \\
\text { ntic } \\
\text { treatmen } \\
t\end{array}$ & $\begin{array}{l}\text { Item 3:I } \\
\text { can } \\
\text { tolerate } \\
\text { the } \\
\text { discomf } \\
\text { ort } \\
\text { during } \\
\text { the } \\
\text { treatme } \\
n t\end{array}$ & $\begin{array}{l}\text { Item } \\
4: I \\
\text { like } \\
\text { to } \\
\text { have } \\
\text { brac } \\
\text { es on } \\
\text { my } \\
\text { teeth }\end{array}$ & $\begin{array}{l}\text { Item 5: } \\
\text { It is } \\
\text { necess } \\
\text { ary for } \\
\text { me to } \\
\text { have } \\
\text { braces }\end{array}$ & \begin{tabular}{|l|} 
Item 1:I \\
am in \\
favor of \\
orthodo \\
ntic \\
treatmen \\
t of my \\
child
\end{tabular} & $\begin{array}{l}\text { Item } 2: I \\
\text { am } \\
\text { willing } \\
\text { to get } \\
\text { orthodo } \\
\text { ntic } \\
\text { treatmen } \\
\text { t of my } \\
\text { child }\end{array}$ & $\begin{array}{l}\text { Item 3: } \\
\text { My } \\
\text { child } \\
\text { can } \\
\text { tolerate } \\
\text { the } \\
\text { discomf } \\
\text { ort } \\
\text { during } \\
\text { the } \\
\text { treatme } \\
\text { nt }\end{array}$ & $\begin{array}{l}\text { Item } \\
4: \text { I } \\
\text { like } \\
\text { to } \\
\text { have } \\
\text { brac } \\
\text { es on } \\
\text { my } \\
\text { child } \\
\text { 's } \\
\text { teeth }\end{array}$ & $\begin{array}{l}\text { Item } \\
\text { 5:It is } \\
\text { necess } \\
\text { ary for } \\
\text { my } \\
\text { child to } \\
\text { have } \\
\text { braces }\end{array}$ \\
\hline $\begin{array}{l}\text { I will } \\
\text { strictly } \\
\text { follow } \\
\text { orthodonti } \\
\text { sts } \\
\text { instruction } \\
\text { s }\end{array}$ & $\begin{array}{l}.343 \\
\text { P } .002\end{array}$ & $\begin{array}{l}.351 \\
\text { P } .002\end{array}$ & $\begin{array}{l}.329 \\
\text { P.003 }\end{array}$ & $\begin{array}{l}.360 \\
\mathrm{P} \\
.001\end{array}$ & $\begin{array}{l}.246 \\
\text { P } .029\end{array}$ & $\begin{array}{l}.241 \\
\text { P } .033\end{array}$ & .208 & .042 & .048 & -.029 \\
\hline $\begin{array}{l}\text { If } \\
\text { orthodonti } \\
\text { sts asked } \\
\text { me to } \\
\text { wear } \\
\text { headgear I } \\
\text { will do as } \\
\text { instructed }\end{array}$ & .21 & $\begin{array}{l}.256 \\
\text { P.023 }\end{array}$ & .167 & .195 & $\begin{array}{l}.390 \\
\mathrm{P}<.01\end{array}$ & .110 & .097 & -.059 & .101 & .120 \\
\hline $\begin{array}{l}\text { If } \\
\text { orthodonti } \\
\text { sts asked } \\
\text { me to } \\
\text { wear } \\
\text { biteplate I } \\
\text { will do as } \\
\text { instructed }\end{array}$ & .092 & .114 & .154 & .132 & .058 & $\begin{array}{l}.252 \\
\text { P } .026\end{array}$ & .169 & .107 & .089 & .014 \\
\hline $\begin{array}{l}\text { If } \\
\text { orthodonti } \\
\text { sts asked } \\
\text { me to } \\
\text { wear } \\
\text { retainers I } \\
\text { will do as } \\
\text { instructed }\end{array}$ & .116 & .091 & .208 & .102 & $\begin{array}{l}.332 \\
\text { P.003 }\end{array}$ & $\begin{array}{l}.331 \\
\text { P.003 }\end{array}$ & $\begin{array}{l}.258 \\
\text { P } .022\end{array}$ & -.203 & .140 & .219 \\
\hline $\begin{array}{l}\text { If } \\
\text { orthodonti } \\
\text { sts asked } \\
\text { me to } \\
\text { wear } \\
\text { elastics I } \\
\text { will do as } \\
\text { instructed } \\
\end{array}$ & .214 & $\begin{array}{l}.295 \\
\text { P } .008\end{array}$ & $\begin{array}{l}.290 \\
\text { P.009 }\end{array}$ & .101 & $\begin{array}{l}.358 \\
\text { P } .001\end{array}$ & .058 & -.068 & -.03 & .108 & -.146 \\
\hline
\end{tabular}




\begin{tabular}{|c|c|c|c|c|c|c|c|c|c|c|}
\hline $\begin{array}{l}\text { Parent } \\
\text { responses } \\
\text { concernin } \\
\text { g } \\
\text { treatment } \\
\text { cooperati } \\
\text { on } \\
\text { My child } \\
\text { will } \\
\text { strictly } \\
\text { follow } \\
\text { orthodonti } \\
\text { st's } \\
\text { instruction } \\
\text { s }\end{array}$ & .052 & .030 & .049 & .176 & .091 & .210 & $\begin{array}{l}.402 \\
\mathrm{P}<.01\end{array}$ & $\begin{array}{l}.289 \\
\text { P .01 }\end{array}$ & .011 & .075 \\
\hline $\begin{array}{l}\text { If } \\
\text { orthodonti } \\
\text { st asked } \\
\text { to wear } \\
\text { headgear } \\
\text { my child } \\
\text { will do as } \\
\text { instructed }\end{array}$ & .117 & .207 & .038 & $\begin{array}{l}.282 \\
\mathrm{P} \\
.012\end{array}$ & $\begin{array}{l}.335 \\
\text { P .001 }\end{array}$ & .191 & $\begin{array}{l}.266 \\
\text { P } .018\end{array}$ & .203 & .085 & $\begin{array}{l}.304 \\
\text { P .006 }\end{array}$ \\
\hline $\begin{array}{l}\text { If } \\
\text { orthodonti } \\
\text { st asked } \\
\text { to wear } \\
\text { biteplate } \\
\text { my child } \\
\text { will do as } \\
\text { instructed }\end{array}$ & $\begin{array}{l}.270 \\
\text { P.016 }\end{array}$ & $\begin{array}{l}.264 \\
\text { P .019 }\end{array}$ & .104 & $\begin{array}{l}.249 \\
\mathrm{P} \\
.027\end{array}$ & $\begin{array}{l}.298 \\
\text { P .008 }\end{array}$ & $\begin{array}{l}.242 \\
\text { P .033 }\end{array}$ & $\begin{array}{l}.362 \\
\text { P.001 }\end{array}$ & $\begin{array}{l}.297 \\
\text { P .008 }\end{array}$ & .129 & $\begin{array}{l}.243 \\
\text { P .031 }\end{array}$ \\
\hline $\begin{array}{l}\text { If } \\
\text { orthodonti } \\
\text { st ask to } \\
\text { wear } \\
\text { retainers } \\
\text { my child } \\
\text { will do as } \\
\text { instructed }\end{array}$ & .072 & .179 & .051 & $\begin{array}{l}.236 \\
\mathrm{P} \\
.036\end{array}$ & $\begin{array}{l}.231 \\
\text { P .041 }\end{array}$ & .104 & $\begin{array}{l}.231 \\
\text { P.041 }\end{array}$ & $\begin{array}{l}.261 \\
\text { P .02 }\end{array}$ & $\begin{array}{l}.241 \\
\mathrm{P} \\
.032\end{array}$ & .216 \\
\hline $\begin{array}{l}\text { If } \\
\text { orthodonti } \\
\text { st asked } \\
\text { to wear } \\
\text { elastics } \\
\text { my child } \\
\text { will do as } \\
\text { instructed }\end{array}$ & .177 & $\begin{array}{l}.290 \\
\text { P .009 }\end{array}$ & .026 & .176 & $\begin{array}{l}.288 \\
\text { P .01 }\end{array}$ & .1 & $\begin{array}{l}.297 \\
\text { P .008 }\end{array}$ & $\begin{array}{l}.281 \\
\text { P .012 }\end{array}$ & .141 & .144 \\
\hline
\end{tabular}

\section{Discussion and Conclusions:-}

Orthodontic attitude of the patients and parents were related significantly when the patients were already in treatment for last 6 months and parents as a whole were found to have higher level of attitude as compared to their children. This correlation obtained during treatment could be due to positive treatment changes appreciable at this time which could have increased their motivation towards treatment. The parental investment (money and time) and 
awareness orpersonal experience probably was the reason for higher parental motivation which is consistent with prior research ${ }^{6,8,9}$.

Significantly higher treatment attitude level of the patients were reported during treatment as the patients were accustomed to wearing treatment appliance and they had felt less discomfort during this time. Prior research showed no variation inlevel of motivation among patients surveyed before treatment and during treatment ${ }^{6}$.

A majority of parents who participated in our survey were mothers or female guardians, indicating that mothers had a higher level of orthodontic concern, which was in consistent with previous findings ${ }^{7,8}$. However, the attitude level of mothers or female guardians was significantly lower after 6 months of orthodontic treatment of their children. Interestingly this was an unexpected result. The female guardians who responded at the beginning of the treatment of their children might have had more expectations from the treatment but till this time the treatment process either did not go as they had expected or they had no time to accompany their children for the follow up appointments. They might not have thought of this aspect of treatment when their children were at the beginning of the treatment. Unlike this, Daniels et al 2009 reported during treatment parents' motivation level was higher than the pretreatment level.

The finding that parents' desire for the children's treatment negatively correlated with the children's desire shows that although parents are willing for the treatment of their children, patients were not actually interested to wear the appliance which shows that wearing braces for patients is a compulsion and parents are the ones who actually seek the treatment for their children ${ }^{7,9,10,11,12}$. Parental encouragement and care especially when their children are in braces could make the children cooperative in the treatment.

Regarding treatment cooperation, we found that higher levels of treatment attitude of the child patients at the beginning of the treatment were correlated with higher levels of their response concerning treatment cooperation such as strictly following orthodontists' instructions, wearing head gear, bite plates, retainers or rubber bands in general. Most literatures report a general dislike for headgear use among orthodontic patients but Adams et al,2009 reported the more motivated the patients are, the more willing they are to cooperate in wearing headgear. The child patients in our study at the beginning of their treatment were unaware of the discomfort they might have when they would be asked to wear headgear,bite plates, retainers or rubber bands. However when the patients were at 6 months treatment stage their response to cooperate with orthodontists' instructions and wearing such appliances was significantly correlated with their treatment attitude response except the response on wearing biteplate. This was because majority of the child patientswith deep bite treated in Tongji Hospital are advised to wear bite plates. And most of them probably did not like to wear the bite plates. On the other hand, only 5 percent of the patients we surveyed had undergone headgear use. When they responded during second time of survey most of them might have already known that they would not be asked to wear headgear and thus their response would not represent those who are actual headgear wearers. The significant correlations we obtained might be because the patients are optimistic for their treatment result and mentally prepared to do whatever they were asked to do by their orthodontists and thus, had an overall positive response regarding treatment cooperation.

Parents' response to orthodontic treatment attitude at the beginning of the treatment was not correlated with the children's treatment cooperationresponse which could be because the parents' judgment about their children's cooperation was not accurate. The psychosocial or physical effects of the appliance on the children were of little concern for their parents. Adams et al,2009 also reported that parents' motivation was not correlated with children's response on treatment cooperation. After 6 months of treatment, however, the parents might have beenquite aware, had encouraged their children to be more cooperative thus orthodontic attitude of parents was then significantly correlated with their children's treatment cooperation responses. Child patients' response regarding treatment attitude correlated significantly with their parents' response on treatment cooperation could be because the parents' had acknowledged the children's motivation to undergo the treatment so that they had been quite sure that their children would then be cooperative. Overall, the orthodontic patients in our study had been good cooperators.

In summary, patients' good cooperation could be anticipated only when orthodontists make an effort to let the patients develop good attitude towards the orthodontic treatment and motivate them. The patients and the parents should be informed clearly the possibility of mild discomfort or pain for few days of appliance placement and activation, necessity of maintaining a good oral hygiene and eating carefully while in braces. Regular talks on the progress of the treatment will also have beneficial behavioral changes. Some patients complain about compromised aesthetics and difficulty eating when they are asked to wear elastics or bite plates, instructions like 'the more you 
cooperate, the faster the result and the earlier the appliance could be removed' may have a positive influence. The decreased parents' level of attitude as the treatment progressed was an unexpected and unfavorable finding. Clinician should motivate parents to be inquisitive about their children's treatment process and to encourage their children to properly follow treatment recommendations. Thus orthodontist should let parentsrealize the behavioral demands of the treatment process and fulfill their responsibility throughout the treatment.

Further survey of Orthodontic Treatment Attitude and Cooperation responses at the end of active treatment and with larger sample size would provide more precise and comprehensive result.

\section{References:-}

1. Adam S. Daniels,Jason D. Seacatand Marita Rohr Inglehart. Orthodontic treatment motivation and cooperation; a cross-sectional analysis of child and adolescent patients' and parents' responses.AJODO,2009, 136: 780-87

2. Brattstrom V, Ingelsson M, Aberg E. Treatment co-operation in orthodontic patients. Br J Orthod 1991;18:3742

3. Cohen L. Social psychological factors associated with malocclusion.

4. Int Dent J 1970; 20:643-53

5. Contemporary Orthodontics, Fourth Edition, William R Proffit, Mosby 2007, 6:167-233

6. ColeWA.Accuracy of patient reporting as an indication of headgear compliance. Am J Orthod Dentofacial Orthop 2002;121:419-23

7. Kreit LH, Burstone C, Delman L. Patient cooperation in orthodontic treatment. J Am Coll Dent 1968; 35:32732

8. MehraT, NandaRS, Sinha PK. Orthodontists' assessment and management of patient compliance. Angle Orthod 1998;68:115-22

9. PietilaT.andPietila I. Parents' views on their own child's dentition compared With an orthodontist's assessment. European Journal of Orthodontics 16 (1994) 309-316

10. Rubin 1998 Behavior modification for orthodontic cooperation, JCO1983May,322-325

11. R.N. Fox, J.E. Albino, L.J. Green, S.D. Farr, and L.A. Tedesco. Development and Validation of a Measure of Attitudes toward Malocclusion. J Dent Res 1982, 61(9):1039-1043

12. Sergl H G, Klages $U$, and Pempera $J$ On the prediction of dentist-evaluated patient compliance in orthodontics. European Journal of Orthodontics 14(1992) 463-468

13. Sergl HG, Klages U, Zentner A. Functional and social discomfort during orthodontic treatment-effects on compliance and prediction of patients' adaptation by personality variables. Eur J Orthod2000; 22:307-15. 